\title{
Cerebrospinal fluid concentrations of soluble CD27 in HTLV-I associated myelopathy and multiple sclerosis
}

\author{
Rogier Q Hintzen, Donald Paty, Joel Oger
}

\begin{abstract}
Objectives-Stimulation of T lymphocytes via the $T$ cell receptor strongly enhances CD27 membrane expression and induces the release of a soluble $32 \mathrm{kDa}$ form of CD27 (sCD27). CD27 is a member of the TNF receptor family, a group of molecules that have important roles in lymphocyte differentiation and survival. Raised concentrations of $\mathrm{SCD} 27$ have been reported in various immunopathological conditions and there is evidence that this molecule can serve as a marker of $T$ cell activation in vivo. Concentrations of sCD27 in CSF were compared between patients with $T$ cell mediated neurological disease and noninflammatory controls. Also, the relation of CSF-sCD27 concentrations with clinical disease activity was investigated in patients with multiple sclerosis.
\end{abstract}

Methods-Four groups were studied: (1) eight patients with HTLV-1 associated myelopathyl tropical spastic paraparisis (HAM)/TSP), (2) eight HTLV-I carriers, (3) 41 patients with multiple sclerosis, and (4) 43 patients with other neurological disease (OND). Concentrations of CSFsCD27 were determined by enzyme linked immunosorbent assay (ELISA).

Results-Quantification of CSF-sCD27 differentiates patients with HAM/TSP from HTLV-I carriers $(p<0.01)$ and from patients with OND $(p<0.001)$. Moreover, the multiple sclerosis patient group was different from the OND group $(p<0.0001)$. In patients with multiple sclerosis, CSFsCD27 concentrations were higher in 24 patients with clinically active disease than in 17 with clinically stable disease. In addition, most of the patients with multiple sclerosis with high sCD27 concentrations showed an increase in EDSS, whereas none of the patients with low sCD27 had an EDSS increase.

Conclusions-As a reliable marker of immunological disease activity in inflammatory white matter disease is still not available, it is proposed that quantification of CSF-sCD27 concentrations is a good candidate. Also, it may serve as a tool to stratify neurological diseases in inflammatory and non-inflammatory states.

(F Neurol Neurosurg Psychiatry 1999;66:791-793)

Keywords: multiple sclerosis, HAM/TSP, T cell activation, TNF-R family

HTLV-I associated myelopathy or tropical spastic paraparesis (HAM/TSP), is an inflammatory demyelinating myelopathy which occurs in occasional (1/400 to $1 / 1000)$ carriers of the HTLV-1 virus. ${ }^{1}$ HAM/TSP shares some clinical and immunopathological characteristics with multiple sclerosis. In multiple sclerosis, demyelination is generally considered to be a result of a breakdown of $\mathrm{T}$ cell tolerance against myelin antigens, whereas in HAM/TSP it has not been ruled out that a direct cytotoxic $\mathrm{T}$ cell response to HTLV-1 is involved in the development of the white matter lesions. ${ }^{2} 3$

In both inflammatory and non-inflammatory neurological disease, most $\mathrm{T}$ cells in CSF express CD27. ${ }^{4}$ CD27 is a member of the TNF-R family, a group of molecules that play important parts in lymphocyte survival and differentiation. ${ }^{5}$ In vitro experiments have shown that activation of $\mathrm{T}$ cells via their TCR-CD3 complex strongly upregulates CD27 expression, predominantly on naive (CD45RA+) cells undergoing transition to memory cells. ${ }^{6}$ Together with this CD27 surface upregulation, a soluble $32 \mathrm{kDa}$ form of CD27 (sCD27 ) is released. ${ }^{7}$ Raised concentrations of sCD27 have been reported in various immunopathological conditions. ${ }^{89}$ Highest concentrations were always seen in compartments where the inflammatory process takes place, such as synovial fluid in arthritis and bronchoalveolar lavage fluid in sarcoidosis. ${ }^{10} 11$ Unlike $\mathrm{T}$ cell derived cytokines, which are sometimes used as markers for immune activation, sCD27 is a stable molecule that can be easily measured in biological fluids. This has motivated us to measure CSF concentrations of sCD27 in HTLV-I carriers and in patients with HAM/TSP as well as in patients with multiple sclerosis and in noninflammatory controls.

Materials and methods

PATIENTS AND SAMPLES

Cerebrospinal fluid was obtained by routine diagnostic lumbar puncture between 1987 and 


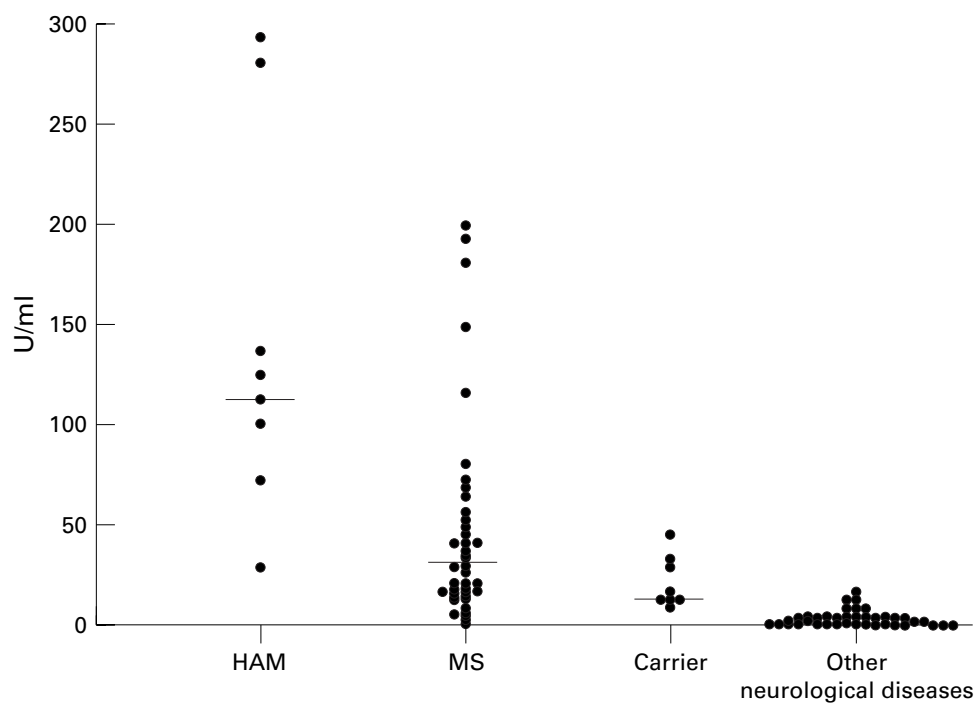

Soluble CD27 in CSF of patients with HAM and controls

Distribution of CSF-sCD27 concentrations expressed as units $/ \mathrm{ml}$ (U/ml) in the different groups: HAM/TSP patients (HAM); patients with multiple sclerosis (MS); HTLV-I carriers; non-inflammatory neurological disease (other). Median values are shown by a horizontal line. fied these 29 patients in two groups: one in which patients had a decline in EDSS of 0.5 point or more during the 3-12 months postlumbar puncture and the second in which patients did not.

ENZYME LINKED IMMUNOSORBENT ASSAY

Soluble CD27 in CSF was quantified with the use of an enzyme linked immunosorbent assay (ELISA ) kit, commercially available from the CLB in Amsterdam, Holland. Ninety six well plates $^{7}$ were coated with a specific CD27 mAb in phosphate buffered saline (CLB-CD27/3). Wells were washed and CSF was added in a fourfold dilution. After overnight incubation at $4^{\circ} \mathrm{C}$, wells were washed again and incubated with a second, biotinylated, specific CD27 $\mathrm{mAb}$ (CLB-CD27/1). A signal was obtained with avidin-peroxidase followed by incubation with tetramethylbenzidin (Merck). Concentrations were arbitrarily expressed as units (U)/ml by reference to a standard curve supplied with the ELISA kit. Normal values for serum with this kit are $<350 \mathrm{U} / \mathrm{ml}$. Tests were performed blind, without knowledge of the clinical diagnosis or clinical state of the patients.

1996 from patients who were seen at the University of British Columbia multiple sclerosis clinic in Vancouver. Samples were aliquoted and stored at $-70^{\circ} \mathrm{C}$. The following groups were studied: (1) patients with HAM/TSP from the British Columbian focus ${ }^{12}(\mathrm{n}=8$, four women, aged 46-76 years, median 58 years), (2) HTLV-I carriers without clinical evidence of neurological disease $(n=8$, six women, aged 30-71 years, median 47 years), (3) patients with clinically definite multiple sclerosis $^{13}$ $(n=41,30$ were relapsing-remitting and 11 were secondary progressive, 23 women, age 20-68 years, median 39 years), (4) patients with other neurological diseases (OND) such as Parkinson's and Alzheimer's disease, fatigue, cerebrovascular accident, myasthenia gravis, diabetic polyneuropathy ( $\mathrm{n}=43,24$ women, age 20-77 years, median 40 years).

Diagnosis was simple among the HTLV-I infected patients: the absence of neurological signs of CNS dysfunction was the criterion for HTLV-I carriers; for HAM/TSP appropriate findings of a progressive myelopathy with inflammatory CSF, pains, spasticity, and early bladder involvement were necessary. ${ }^{1}$

Correlation between CSF-sCD27 and clinical disease activity was investigated in two ways.

In a first approach, disease activity was defined by the presence of new or recurrent symptoms (lasting more than 24 hours) that were seen at the time that the lumbar puncture was performed or at the next visit within 3 months postlumbar puncture. Using this set of criteria, 24 patients had active multiple sclerosis and 17 patients had stable disease. In the second approach we identified 29 patients who were part of our systematic follow up system recorded using Costar ${ }^{14}$ and had repeated evaluation with the Kurtzke extended disability status score (EDSS) after the lumbar puncture. The remaining 12 patients had not been included in this systematic follow up. We strati-
STATISTICS

An unpaired $t$ test was used to compare CSFsCD27 concentrations between the different patient groups. A $\chi^{2}$ test was applied for the testing of the relation between EDSS and CSF-sCD27 in multiple sclerosis.

\section{Results}

CSF-SCD27 CONCENTRATIONS IN DIFFERENT

GROUPS

Results for the four groups are shown in the figure. The highest concentrations of sCD27 (mean (SEM)) were found in HAM/TSP: 143 (34) $\mathrm{U} / \mathrm{ml}$ (range 28-293). This was significantly higher $(\mathrm{p}<0.01)$ than for the HTLV-I carriers: 19 (4) $\mathrm{U} / \mathrm{ml}$ (range 4-44); there was only one overlapping value. These concentrations (3 (1); $<<0.00005$, range 4-16). Moreover, the 41 patients with multiple sclerosis (46 (6)) showed significantly higher values $(p<0.001)$ than the OND patients but there was some overlap between these two groups (multiple sclerosis range $0-198 \mathrm{U} / \mathrm{ml}$ ). All values in the OND group were $<16 \mathrm{U} / \mathrm{ml}$, so this value of 16 $\mathrm{U} / \mathrm{ml}$ was used as a cut off for normality of CSF concentrations.

RELATION WITH CLINICAL DISEASE ACTIVITY IN MULTIPLE SCLEROSIS

There was no difference between concentrations of CSF-sCD27 in patients with relapsingremitting $v$ patients with secondary progressive multiple sclerosis. The group of patients with clinically active multiple sclerosis at the time of lumbar puncture had an sCD27 concentration of $66(11) \mathrm{U} / \mathrm{ml}(\mathrm{n}=24) v 17(4) \mathrm{U} / \mathrm{ml}$ for the group of patients $(n=17)$ with clinically inactive disease $(p<0.004)$. From this group of 41 patients, data on repeated EDSS were available for 29 patients. Sixteen of them had no EDSS increase over time after lumbar puncture; their values were 19 (5) $\mathrm{U} / \mathrm{ml}$, range 0 to 72 . The were also higher than those of the OND group: 
High CSF-sCD27 in patients with multiple sclerosis is associated with decline in EDSS

\begin{tabular}{llll}
\hline & $s C D 27 \leqslant 16 \mathrm{U} / \mathrm{ml}$ & $s C D 27>16 \mathrm{U} / \mathrm{ml}$ & Total \\
\hline No EDSS decline & 10 & 6 & 16 \\
EDSS decline & 0 & 13 & 13 \\
Total & 10 & 19 & 29 \\
\hline
\end{tabular}

other 13 that did show an increase of their EDSS had significantly higher values: 72 (15), range $20-198(\mathrm{p}<0.001)$.

In addition, the table shows that 19 out of the 29 patients with available EDSS course had CSF-sCD27 values above the maximum of the OND population $(>16 \mathrm{U} / \mathrm{ml})$. From these 19 patients, 13 had an increase in EDSS over time, whereas none of the 10 paients with CSFsCD27 values equal or below $16 \mathrm{U} / \mathrm{ml}$ had an increase in EDSS.

Some (but not all) patients with HTLV-I had serum available for testing. Patients with HAM/TSP ( $n=6$, mean (SEM) 518 (116), range 360-1080) were not significantly higher than carriers ( $n=9,306$ (158), range 108-560). Both groups had higher serum values than those found in samples of healthy subjects $(n=9)$ which were all $<250 \mathrm{U} / \mathrm{ml}$. We did not measure sCD27 in serum of patients with multiple sclerosis as we have previously shown that it is not different from controls. ${ }^{8}$

\section{Discussion}

This study confirms earlier findings that concentrations of the $\mathrm{T}$ cell activaton marker sCD27 are raised in the CSF of patients with multiple sclerosis when compared with patients with non-inflammatory disease. ${ }^{8}$ In addition, we have shown that CSF-sCD27 concentrations are higher in patients with HAM/TSP than in HTLV-1 carriers or patients with multiple sclerosis. A difference in CSF concentrations of sCD27 between multiple sclerosis and $\mathrm{HAM}$ /TSP groups could be related to a higher and more chronic state of immune activation in the latter disease, although a difference in localisation of the inflammatory lesions in either disease could also contribute to this. ${ }^{15}$ Interestingly, HTLV-I carriers also had slightly higher concentrations of CSF-sCD27 than the OND group $(p<0.01)$. This suggests that in these neurologically asymptomatic subjects a low level of intrathecal immune activation already takes place. Indeed, some small white matter lesions are often seen on MRI of HTLV-I carriers ${ }^{16}$ (personal observation), although the histopathological correlate for these lesions is thus far unknown.

Serum concentrations of sCD27 in patients with HAM/TSP and HTLV-I carriers showed a wide range, from 110 to $1080 \mathrm{U} / \mathrm{ml}$. Many patients had higher concentrations than the normal values for healthy controls. ${ }^{8}$ We suspect that this is a reflection of the different concomitant systemic pathologies often associated with HTLV-I infection, such as arthritis or bronchoalveolitis. ${ }^{1}$ As the HAM/TSP samples of CSF were not paired in time, we could not directly show that $\mathrm{sCD} 27$ is secreted intrathecally, However, we have previously shown that it was very unlikely that the presence of sCD27 in CSF would result from leakage through the blood-brain barrier. ${ }^{8}$

In this study, CSF sCD27 concentrations in multiple sclerosis also correlated with clinical activity. We think that this finding is worth reporting as it may reflect immunological disease activity. In multiple sclerosis, clinical activity underreflects actual disease activity, the latter being better evaluated with MRI. It is of note here that most but not all (13 out of 19) of the patients with high sCD27 (supposed immunological disease activity) showed an increase in EDSS, whereas none of the 10 patients with low CD27 had an EDSS increase.

Although an increase of sCD27 in CSF is by no means specific for demyelinating disease, ${ }^{8}$ the differences described here in sCD27 concentrations might be of practical benefit and provide an easy tool to differentiate between inflammatory and non-inflammatory neurological disease. Moreover, this assay might help to differentiate patients with HAM/ TSP from those HTLV-I carriers who have neurological dysfunction due to other aetiologies such as cervicarthrosis or vascular disease.

$\mathrm{RQH}$ was on study leave from the Department of Neurology, Leiden University Medical Centre, The Netherlands, and was supported by a travel grant from the Stichting Vrienden multiple sclerosis Research Netherlands. This work was supported by the Christopher Foundation (Vancouver). Tariq Aziz is gratefully acknowledged for his excellent technical assistance. This work acknowledged for his excellent technical assistance. This work
was presented in part at the 50th meeting of the AAN in Minnewas presented in part at the 50 th meeting of the AAN in Min

1 Gessain A, Gout O. Chronic myelopathy associated with human T-lymphotropic virus I (HTLV-I). Ann Intern Med 1992;117:933-46

2 Jacobson S, McFarlin DE, Robinson S, et al. HTLV-I specific cytotoxic T-lymphocytes in the cerebrospinal fluid of patients with HTLV-I-associated neurological disease. Ann Neurol 1992;32:651-7.

3 Oger J, Dekaban GA. HTLV-I Associated myelopathy: a case of viral-induced autoimmunity. Autoimmunity 1995; 21:151-9.

4 Hintzen RQ, Fiszer U, Fredrikson S, et al. Analysis of CD27 surface expression on $\mathrm{T}$ cell subsets in MS patients and control individuals. F Neuroimmunol 1996;56:99-105.

5 Hintzen RQ, de Jong R, van Lier RAW. CD27: marker and mediator of T-cell activation. Immunol Today 1994;15:30711.

6 Hintzen RQ, de Jong R, Lens SM, et al. Regulation of CD27 expression on subsets of mature T-lymphocytes. F Immunol 1993;151:2426-35.

7 Hintzen RQ, de Jong R, Hack CE, et al. A soluble form of the human T-cell differentiation antigen CD27 is released after triggering

1991;147:29-35.
8 Hintzen RQ, van Lier RAW, Kuijpers KC, et al. Elevated levels of a soluble form of the T-cell activation antigen CD27 in cerebrospinal fluid of multiple sclerosis patients. $\mathcal{F}$ Neuroimmunol 1991;35:211-17.

9 Font J, Pallares L, Martorell J, et al. Elevated soluble CD27 levels in serum of patients with systemic lupus erythematosus. Clin Immunol Immunopathol 1996;81:239-43.

10 Tak PP, Hintzen RQ, Teunissen JJ, et al. Expression of the activation antigen CD27. Clin Immunol Immunopathol 1996;80:129-38.

11 Hol BE, Hintzen RQ, Van Lier RA, et al. Soluble and cellular markers of $\mathrm{T}$ cell activation in patients with pulmonary sarcoidosis. Am Rev Respir Dis 1993;148:643-9.

12 Oger J, Werker DH, Foti DJ, et al. HTLV-I associated myelopathy: an endemic disease of Canadian Aboriginals of the northwest Pacific. Can f Neurol Sci 1993;20:302-6.

13 Poser CM, Paty DW, Scheinberg LC, et al. New diagnosistic critera for MS: guidelines for research protocols. Ann Neuol 1983;13:227-31.

14 Paty, D, Studney D, Redekop K, et al. A computerized patient record adapted for clinical research purposes. Ann Neurol 1994;36(suppl):S134-5.

5 Rudge P, Ali A, Cruickshank JK. Multiple sclerosis, tropical spastic paraparesis and HTLV-I infection in AfroCaribbean patients in the United Kingdom. F Neurol Neusosurg Psychiatry 1991;54:689-94.

16 Kira JI, Fujihara K, Itoyama Y, et al. Leukoencephalopathy in HTLV-I associated myelopathy/tropical spastic fter corticosteroid therapy. F Neurol Sci 1991;106:41-9. 